\title{
Study of Essential Norms and Standards for Electrical and Electroni- cal Experiment Teaching
}

\author{
Rajesh Kumar, Peter S. Nasr \\ Department of Biochemistry, Academy of Sciences, Technology and Research, Canada
}

Abstract: To realize the teaching goals in cultivating students'ability of conducting self-study,freely implementing of knowl- edge,developing scientific research skills,establishing team collaborative spirit,nurturing the innovative capacity and comprehensive quality,four universities led by Southeast University have conducted joint survey and design. Finally,we formulate the essential experi- ment teaching norms and standards regarding the required knowledge for experiment,experiment skills,technical methods for experi- ment,practice capacity and comprehensive qualification. The norms and standards have proven to generate fruitful and positive effects on experiment teaching.

Keywords: Electrics and electronics; experiment teaching; teaching norms and standards

The experimental teaching of Electrotechnics and electronics basic course is oriented to electrical engineering, electronic information, automation, computer and other specialties. It has the characteristics of serving a large group of students, cooperating with many theoretical courses and teaching hours of experimental teaching.

However, the current electrician and electronic experiment teaching lacks the system integrity, the clear detailed experiment teaching standard request, the teaching lacks the instruction norm rule. Most of the teachers based on their own understanding of the teaching objectives, organized to carry out teaching; teachers lack of comprehensive and systematic learning ways, mainly rely on their own experience accumulation and mutual exchange; students in learning and practice to obtain experimental basic knowledge, In the open and autonomous teaching practice, students can not get timely and effective guidance when they encounter problems.

A research group composed of the national and provincial electrician and electronic experimental teaching demonstration centers of Southeast University, Nanjing University of posts and Telecommunications, Nanjing normal University and Nanjing Institute of Engineering, in order to train students to study independently and carry out practical projects, The aim of exploring and innovating comprehensive ability quality is to develop it from several aspects, such as the basic teaching requirement, the teaching implementation scheme, the construction of environmental conditions, and so on.

The research and design of the basic standard of electrical and electronic experiment teaching.

\section{Subject research method}

The schools of the members of the research group are the comprehensive universities with the characteristics of engineering, the universities of engineering majoring in electricity, the comprehensive universities of arts and science, and the universities of applied undergraduate, etc. The training items have their own characteristics. The research group makes full use of its own advantages, collects widely statistical teaching objectives and training requirements, and develops the practice of teaching and learning.

Copyright (C) 2018 Rajesh Kumar et al.

doi: $10.18063 /$ peee.v1i2.

This is an open-access article distributed under the terms of the Creative Commons Attribution Unported License

(http://creativecommons.org/licenses/by-nc/4.0/), which permits unrestricted use, distribution, and reproduction in any medium, provided the original work is properly cited. 


\subsection{Collect samples of teaching needs}

According to the teaching requirements of electrical engineering and electronic information specialties in different types and different levels of schools at the level of basic courses ${ }^{[1]}$, according to the requirements of electrical engineering and electronic information industry for personnel training specifications ${ }^{[2]}$, according to the requirements of engineering education professional certification, according to the experimental teaching training Students'basic abilities and accomplishments of self-study, knowledge application, scientific research, engineering practice, teamwork, and exploration and innovation enable them to learn to study independently, learn to do learning, learn to research and explore and learn to design innovative goals in practice, and widely collect requirements and objectives from all aspects.

\subsection{Basic standards of design teaching}

According to the sample information collected, combined with the current educational ideas, teaching system contents, the transformation and renewal of teaching technology and methods, statistics, induction, summary and formation of experimental knowledge, experimental skills, technical methods, practical ability, Comprehensive quality of five aspects of the development of experimental teaching comprehensive, clear, detailed basic standard of the subject research objectives $^{[3]}$.

\subsection{Designing teaching facilities}

The purpose of establishing teaching standard is to use the standard to guide teaching content setting, teaching process design, teaching mode reform, examination mechanism construction and so on in order to achieve the basic goal of experimental teaching. The project group designed a whole

The system of electrician and electronic experiment demonstration teaching is applied in their respective schools, and the basic teaching standards are adjusted and perfected in teaching practice.

\subsection{Summarize the research results}

Through the teaching practice test, the paper summarizes and condenses, forms the elementary achievement of the basic standard of teaching, and puts forward the requirements of the practice environment.

\section{Basic standards of experimental teaching}

After more than two years of research and practice, the formation of experimental knowledge, experimental skills, technical methods, The basic standard of electrician and electronic experiment teaching is composed of the index system of 130 detailed rules of 38 major categories and 5 aspects of practical ability and comprehensive quality. As shown in tables 1 to 5 .

\begin{tabular}{|c|c|c|c|}
\hline \multirow{7}{*}{\multicolumn{2}{|c|}{$\begin{array}{l}\text { experiment speci- } \\
\text { fication }\end{array}$}} & $\begin{array}{l}\text { Power generation and } \\
\text {-transmission }\end{array}$ & $\begin{array}{l}\text { Electrical energy generation, power transmission, voltage level, electrical } \\
\text { equipment and facilities }\end{array}$ \\
\hline & & Electricity safety knowledge & Power supply facilities and circuits in experimental sites \\
\hline & & & Safety voltage, safe current, electric shock prevention and rescue \\
\hline & & & Grounding of electrical equipment, shielding \\
\hline & & $\begin{array}{l}\text { Experimental } \\
\text { specification }\end{array}$ & Access to laboratory requirements, equipment access, safety and hygiene \\
\hline & & & Preview, booking, allocation, acceptance, examination \\
\hline & & & Equipment operation, circuit test, electrification test \\
\hline
\end{tabular}




\begin{tabular}{|c|c|c|}
\hline \multicolumn{3}{|c|}{ Electronic elementDevice recognition } \\
\hline \multirow[t]{4}{*}{ characteristics } & characteristic narameter & \multirow{4}{*}{$\begin{array}{l}\text { Resistors, potentiometers, capacitors, inductors, diodes, Triode MOS transis- } \\
\text { tors, keys, switches, displays }\end{array}$} \\
\hline & & \\
\hline & parameter measurement & \\
\hline & Application characteristics & \\
\hline \multirow{4}{*}{$\begin{array}{l}\text { Structure and Ap- } \\
\text { plication of Elec- } \\
\text { trical equipment }\end{array}$} & structure function & \multirow{4}{*}{$\begin{array}{l}\text { Contactors, buttons, fuses, autotransformer, relays, delay relays, DC motors, } \\
\text { AC three-phase motors }\end{array}$} \\
\hline & Parameter specification & \\
\hline & Application condition & \\
\hline & Application characteristics & \\
\hline \multirow{5}{*}{$\begin{array}{l}\text { Object and method } \\
\text { of measurement }\end{array}$} & Electric energy parameter & Current, voltage, power \\
\hline & Electrical signal feature & Waveform, amplitude, frequency, phase, noise logic state \\
\hline & circuit parameter & I / O impedance, quality factor, device parameters, gain, distortion \\
\hline & characteristic curve & $\begin{array}{l}\text { Transmission characteristic, volt-ampere characteristic, frequency characteris- } \\
\text { tic, load characteristic }\end{array}$ \\
\hline & measuring method & $\begin{array}{l}\text { Direct measurement, indirect measurement, combined measurement, compara- } \\
\text { tive measurement }\end{array}$ \\
\hline
\end{tabular}

Table 1. Experimental knowledge

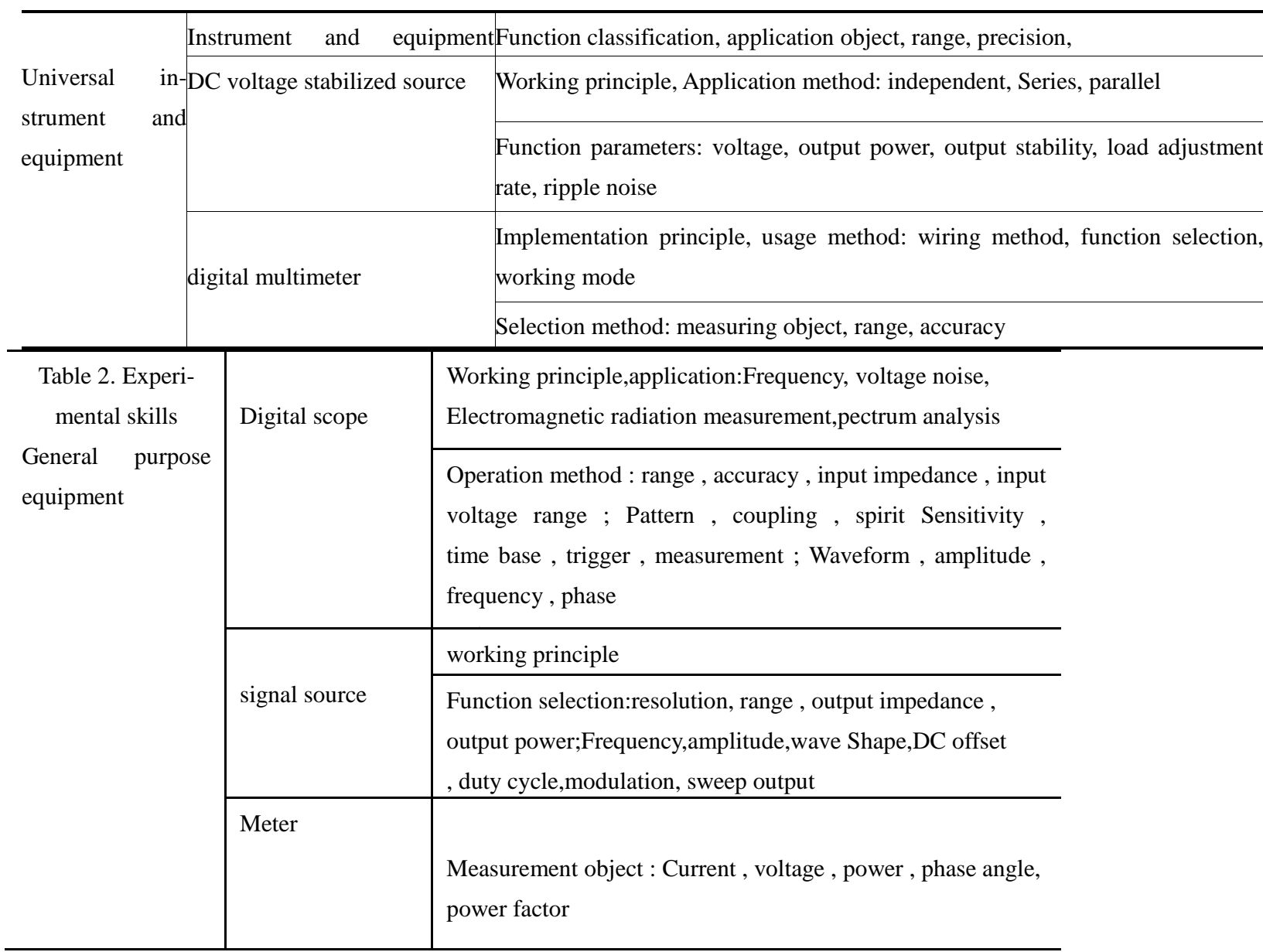




\begin{tabular}{|c|c|c|}
\hline \multirow[t]{6}{*}{$\begin{array}{l}\text { dedicated } \\
\text { ment }\end{array}$} & $\begin{array}{l}\text { Transistor char- } \\
\text { acteristics Tester }\end{array}$ & $\begin{array}{l}\text { Diode, Zener, Bipolar Transistor, Field Effect Tube Char- } \\
\text { acteristics Test }\end{array}$ \\
\hline & logic analyzer & Sampling trigger mode, storage depth \\
\hline & Digital sweeper & $\begin{array}{l}\text { Passive RCL Network,active amplifier amplitude-frequency } \\
\text { characteristics,phase-frequency characteristics test }\end{array}$ \\
\hline & High voltage & Measuring range, measurement accuracy \\
\hline & $\begin{array}{l}\text { Programmable } \\
\text { voltage source }\end{array}$ & $\begin{array}{l}\text { Voltage range, current output capability, voltage accuracy, } \\
\text { voltage stability, ripple }\end{array}$ \\
\hline & $\begin{array}{l}\text { Spectrum Ana- } \\
\text { lyzer }\end{array}$ & $\begin{array}{l}\text { Measuring range, center frequency, frequency resolution ; } \\
\text { Tracking source }\end{array}$ \\
\hline \multirow[t]{2}{*}{ Cable } & \multirow[t]{2}{*}{ Cable category } & $\begin{array}{l}\text { Single-strand hard wire, multi-strand flexible wire, shielded } \\
\text { cable }\end{array}$ \\
\hline & & Cable impedance characteristics and applications \\
\hline \multirow[t]{2}{*}{ Connector } & $\begin{array}{l}\text { Classification and } \\
\text { characteristics }\end{array}$ & BNC,DIP Socket,banana plug,phoenix terminal,air connector \\
\hline & use & $\begin{array}{l}\text { Power connection ( current ), circuit connection ( lap } \\
\text { mode ), channel connection ( Signal strength , frequency } \\
\text { Range, impedance matching ) }\end{array}$ \\
\hline \multirow[t]{3}{*}{$\begin{array}{l}\text { design Simulation } \\
\text { software }\end{array}$} & $\begin{array}{l}\text { Application soft- } \\
\text { ware classifica- } \\
\text { tion }\end{array}$ & \multirow[t]{3}{*}{$\begin{array}{l}\text { Spice, Multisim, Matlab, Protel, Labview , FilterPro, } \\
\text { SwitchPro Wait }\end{array}$} \\
\hline & Basic use & \\
\hline & Application skills & \\
\hline \multirow[t]{5}{*}{ Common circuit } & Signal generation & $\begin{array}{l}555 \text { Oscillator,Venturi oscillator circuit,crystal oscillator cir- } \\
\text { cuit, }\end{array}$ \\
\hline & Signal conversion & $\begin{array}{l}\text { Voltage and current conversion, isolation coupling, voltage } \\
\text { divider } \\
\text {, current limit, V / F , F / V , ADC, DAC, difference Points / } \\
\text { Common mode, impedance conversion, zero-crossing com- }\end{array}$ \\
\hline & signal input & $\begin{array}{l}\text { Switch input, press button lose Into, elimination shake Dy- } \\
\text { namic and electric level turn Change, logic Series Electricity } \\
\text { Flat and poor Minute letter Number, Bridge }\end{array}$ \\
\hline & Display circuit & Status display, data display, curve display \\
\hline & Drive circuit & $\begin{array}{l}\text { Voltage amplification, current drive, LED Drive, relay } \\
\text { drive, DC motor drive, step Into motor drive, audio power } \\
\text { drive, digital tube drive }\end{array}$ \\
\hline
\end{tabular}




\begin{tabular}{|c|c|c|}
\hline \multirow[t]{3}{*}{$\begin{array}{l}\text { Voltage } \\
\text { source selection }\end{array}$} & $\begin{array}{l}\text { Voltage source } \\
\text { circuit }\end{array}$ & $\begin{array}{l}\text { Linear DC voltage source, series regulator circuit, } \\
\text { integrated voltage regulator circuit, switching power supply } \\
\text {, photovoltaic power supply }\end{array}$ \\
\hline & $\begin{array}{l}\text { Voltage source } \\
\text { Main characteris- } \\
\text { tic parameter }\end{array}$ & \multirow[t]{2}{*}{$\begin{array}{l}\text { Voltage stability, voltage accuracy, ripple, load regulation, } \\
\text { voltage regulation }\end{array}$} \\
\hline & $\begin{array}{l}\text { Use of voltage } \\
\text { source }\end{array}$ & \\
\hline \multirow{12}{*}{$\begin{array}{l}\text { Table 2. (Contin- } \\
\text { ued) }\end{array}$} & \multirow{2}{*}{$\begin{array}{l}\text { Linear component } \\
\text { Instrument and } \\
\text { equipment charac- } \\
\text { teristics }\end{array}$} & R , C , L Component volt-ampere and frequency characteris- \\
\hline & & $\begin{array}{l}\text { Scope of application, measurement object and range accura- } \\
\text { cy }\end{array}$ \\
\hline & $\begin{array}{l}\text { Circuit law appli- } \\
\text { cation }\end{array}$ & $\begin{array}{l}\text { Circuit Theorem Verification Circuit Design , Parametric } \\
\text { Testing and Verification }\end{array}$ \\
\hline & $\begin{array}{l}\text { Passive network } \\
\text { characteristics }\end{array}$ & $\begin{array}{l}\text { Passive dual port network volt-ampere and frequency charac- } \\
\text { teristics }\end{array}$ \\
\hline & $\begin{array}{l}\text { Active network } \\
\text { characteristics }\end{array}$ & $\begin{array}{l}\text { Active network equivalent analysis and volt-ampere , fre- } \\
\text { quency characteristic test }\end{array}$ \\
\hline & $\begin{array}{l}\text { Resonance circuit } \\
\text { research }\end{array}$ & Resonance realization condition and quality factor \\
\hline & $\begin{array}{l}\text { Controlled source } \\
\text { circuit }\end{array}$ & $\begin{array}{l}\text { Controlled source feature analysis, implementation method } \\
\text { and effective range }\end{array}$ \\
\hline & $\begin{array}{l}\text { Voltage source } \\
\text { characteristics }\end{array}$ & $\begin{array}{l}\text { Accuracy, stability, power, noise, voltage regulation, load } \\
\text { regulation, power Pressure source implementation method }\end{array}$ \\
\hline & $\begin{array}{l}\text { Current source } \\
\text { characteristics }\end{array}$ & Accuracy, stability, voltage range \\
\hline & AC circuit test & AC circuit current, voltage, power measurement and analy- \\
\hline & $\begin{array}{l}\text { Power factor and } \\
\text { its djustment }\end{array}$ & Power factor adjustment method and adjustment degree \\
\hline & AC circuit control & AC circuit design, implementation and commissioning \\
\hline \multirow{2}{*}{$\begin{array}{l}\text { Analog } \\
\text { Design }^{[5]}\end{array}$} & $\begin{array}{l}\text { Diode characteris- } \\
\text { tics and applica- }\end{array}$ & $\begin{array}{l}\text { Rectification, detection, voltage regulation, illumination, } \\
\text { variable volume }\end{array}$ \\
\hline & $\begin{array}{l}\text { Triode circuit asic } \\
\text { parameters }\end{array}$ & $\begin{array}{l}\text { Static operating point adjustment,input and output imped- } \\
\text { ance,signal distortion cause analysis,transmission } \\
\text { Sex,frequency characteristics }\end{array}$ \\
\hline
\end{tabular}




\begin{tabular}{|c|c|c|c|}
\hline & & $\begin{array}{l}\text { Transistor charac- } \\
\text { teristics and Typi- } \\
\text { cal application } \\
\text { circuit }\end{array}$ & $\begin{array}{l}\text { Common circuit, common-emitter circuit, common-base } \\
\text { circuit application characteristics }\end{array}$ \\
\hline & & $\begin{array}{l}\text { FET characteristics } \\
\text { and Typical appli- }\end{array}$ & $\begin{array}{l}\text { Common source circuit, common drain circuit, common gate } \\
\text { circuit application characteristics }\end{array}$ \\
\hline & & $\begin{array}{l}\text { Differential ampli- } \\
\text { fier circuit }\end{array}$ & $\begin{array}{l}\text { Differential mode common mode signal, basic differential } \\
\text { amplifier circuit characteristics, current source circuit }\end{array}$ \\
\hline & & $\begin{array}{l}\text { Multi-level feed- } \\
\text { back amplification } \\
\text { circuit design }\end{array}$ & $\begin{array}{l}\text { Multi-stage amplifier function setting, input impedance de- } \\
\text { sign, } \\
\text { gain distribution, output power setting Meter, feedback de- }\end{array}$ \\
\hline & & $\begin{array}{l}\text { Power amplifica- } \\
\text { tion }\end{array}$ & $\begin{array}{l}\text { Class A and Class B amplifiers, C and D Class power ampli- } \\
\text { fier.set success. etc. }\end{array}$ \\
\hline & & $\begin{array}{l}\text { Signal generation } \\
\text { and conversion }\end{array}$ & $\begin{array}{l}\text { Signal generation and sine wave, pulse wave, triangle wave, } \\
\text { pulse wave conversion }\end{array}$ \\
\hline & & $\begin{array}{l}\text { Operational ampli- } \\
\text { fier basic applica- }\end{array}$ & $\begin{array}{l}\text { Co-directional, reverse proportional amplification, basic } \\
\text { arithmetic circuit, comparator, detection rectification }\end{array}$ \\
\hline & & Op amp gain con- & Multiple gain control methods \\
\hline & & $\begin{array}{l}\text { Multi-stage opera- } \\
\text { tional amplifier } \\
\text { circuit design }\end{array}$ & $\begin{array}{l}\text { Input and output impedance, impedance matching, gain } \\
\text { distribution, bandwidth, bandwidth gain product, band In- } \\
\text { ternal gain fluctuation }\end{array}$ \\
\hline & & $\begin{array}{l}\text { Filter circuit de- } \\
\text { sign }\end{array}$ & $\begin{array}{l}\text { Low pass, high pass, band pass, band stop filter ; Mul- } \\
\text { ti-order, high-order filter, with internal wave Dynamic and } \\
\text { out-of- band attenuation }\end{array}$ \\
\hline & & $\begin{array}{l}\text { Linear power sup- } \\
\text { ply design and } \\
\text { implementation }\end{array}$ & $\begin{array}{l}\text { Buck, rectification, filtering, voltage regulation, voltage } \\
\text { regulation }\end{array}$ \\
\hline & & $\begin{array}{l}\text { DC/DC Voltage } \\
\text { conversion circuit }\end{array}$ & $\begin{array}{l}\text { Rise / Buck, isolation / Non-isolated circuit basic configura- } \\
\text { tion, input voltage range, output power Pressure control }\end{array}$ \\
\hline \multirow{5}{*}{\multicolumn{2}{|c|}{$\begin{array}{l}\text { digital } \\
\text { circuit }\end{array}$}} & $\begin{array}{l}\text { Gate characteris- } \\
\text { tics }\end{array}$ & $\begin{array}{l}\text { Level and voltage, sink current / Pull out current, rise and } \\
\text { fall edge time, door power Road delay, output voltage and } \\
\text { current relationship }\end{array}$ \\
\hline & & \multirow{3}{*}{$\begin{array}{l}\text { Combinational } \\
\text { logic design }\end{array}$} & $\begin{array}{l}\text { Driver , buffer, tri-state gate characteristics and uses, level } \\
\text { Shifting, }\end{array}$ \\
\hline & & & $\begin{array}{l}\text { Logical combination,conversion,coding / Decoding,logic } \\
\text { operation circuit, }\end{array}$ \\
\hline & & & Logical operation,simplification \\
\hline & & $\begin{array}{l}\text { Sequential logic } \\
\text { design }\end{array}$ & $\begin{array}{l}\text { Trigger,shift register,counter,variable range counter,variable } \\
\text { step counter }\end{array}$ \\
\hline
\end{tabular}




\begin{tabular}{|c|c|c|}
\hline $\begin{array}{l}\text { Hybrid logic de- } \\
\text { sign }\end{array}$ & \multicolumn{2}{|c|}{ Calculator, state machine, controller, memory } \\
\hline \multirow{4}{*}{$\begin{array}{l}\text { Table } 3 . \\
\text { Technical } \\
\text { method } \\
\text { Digital mod- } \\
\text { el Hybrid } \\
\text { circui }\end{array}$} & $\begin{array}{l}\text { Analog to digital } \\
\text { converter ADC }\end{array}$ & $\begin{array}{l}\text { Conversion mode, conversion speed, conversion accuracy, } \\
\text { input voltage range, reference voltage }\end{array}$ \\
\hline & $\begin{array}{l}\text { Digital to analog con- } \\
\text { verter DAC }\end{array}$ & $\begin{array}{l}\text { Conversion speed, conversion accuracy, output mode } \\
\text { ( Voltage / Current ) The output voltage / Electricity Flow } \\
\text { range }\end{array}$ \\
\hline & Gain control & $\begin{array}{l}\text { Digital potentiometer, analog switch switching resistor, } \\
\text { relay switching resistor }\end{array}$ \\
\hline & Voltage control & $\begin{array}{l}\text { Digital potentiometer, analog switch switching resistor, } \\
\text { relay switching resistor }\end{array}$ \\
\hline \multirow{3}{*}{$\begin{array}{l}\text { FPGA } \\
\text { application }\end{array}$} & $\begin{array}{l}\text { Hardware description } \\
\text { language design }\end{array}$ & VHDL, Verilog \\
\hline & $\begin{array}{l}\text { Design simulation soft- } \\
\text { ware use }\end{array}$ & Quartus, ISE \\
\hline & $\begin{array}{l}\text { Digital system design } \\
\text { method }\end{array}$ & $\begin{array}{l}\text { System structure design , top-down, bottom-up design } \\
\text { method, human-computer interaction channel design }\end{array}$ \\
\hline \multirow[t]{6}{*}{$\begin{array}{l}\text { electronic } \\
\text { system de- } \\
\text { sign }\end{array}$} & $\begin{array}{l}\text { Electronic system design } \\
\text { method }\end{array}$ & Electronic system structure, system design method \\
\hline & \multirow{2}{*}{$\begin{array}{l}\text { Microprocessor selection } \\
\text { and application }\end{array}$} & MCU, embedded processor , DSP \\
\hline & & $\begin{array}{l}\text { Digits, frequency, command system, IO Interface, power } \\
\text { consumption, memory configuration, debugging sur- } \\
\text { roundings }\end{array}$ \\
\hline & $\begin{array}{l}\text { Sensor characteristics } \\
\text { and detection circuit }\end{array}$ & $\begin{array}{l}\text { Temperature, humidity, illuminance, gas, weight, speed, } \\
\text { acceleration, position, position Shift, angle, electric field, } \\
\text { magnetic field sensor and detection method thereof }\end{array}$ \\
\hline & Actuator and its driver & $\begin{array}{l}\text { Voltage amplification , power drive , motor control, } \\
\text { electromagnetic mechanism control }\end{array}$ \\
\hline & $\begin{array}{l}\text { Human-computer inter- } \\
\text { action channel }\end{array}$ & $\begin{array}{l}\text { Buttons, switches, knobs, dials, touch screens ; Indicator } \\
\text { light, digital tube display, } \\
\text { LCD, vibration, sound }\end{array}$ \\
\hline \multirow{3}{*}{$\begin{array}{l}\text { Table 3. ( Con- } \\
\quad \text { tinued ) } \\
\text { experimental } \\
\text { design }\end{array}$} & Basic Information & $\begin{array}{l}\text { Course name, experiment name, time, location, class, } \\
\text { student number }\end{array}$ \\
\hline & $\begin{array}{l}\text { Experimental princi- } \\
\text { ple }\end{array}$ & Theoretical basis, experimental ideas \\
\hline & Experimental program & $\begin{array}{l}\text { Implementation method, experimental steps, expected } \\
\text { goals }\end{array}$ \\
\hline
\end{tabular}




\begin{tabular}{|c|c|c|}
\hline & Circuit design & Circuit design, component selection, simulation optimiza- \\
\hline & $\begin{array}{l}\text { Measurement } \\
\text { methods }\end{array}$ & $\begin{array}{l}\text { Measuring method, measuring instrument, measuring cir- } \\
\text { instrument selection }\end{array}$ \\
\hline & Experimental process & Experimental procedure, operation, measurement sequence \\
\hline & data record & Data table design, data record \\
\hline & Result analysis & Data processing, error analysis, performance analysis \\
\hline \multirow{5}{*}{ Circuit design } & Circuit selection & Typical application circuit structure model selection \\
\hline & Circuit design & Circuit modification, homemade design \\
\hline & Device selection & $\begin{array}{l}\text { Specifications, parameters, accuracy, power, material, } \\
\text { withstand voltage, current, package }\end{array}$ \\
\hline & Module selection & Existing device, circuit, module selection \\
\hline & Matching parameter & Input and output parameters \\
\hline \multirow{2}{*}{$\begin{array}{l}\text { Circuit imple- } \\
\text { mentation }\end{array}$} & Way of realization & Breadboard, universal orifice, printed circuit board \\
\hline & Implementation & $\begin{array}{l}\text { Component selection, circuit layout, component plug-in, } \\
\text { wire bonding }\end{array}$ \\
\hline \multirow[t]{3}{*}{ Debug test } & Debugging method & $\begin{array}{l}\text { Unit circuit debugging, module debugging, cascade de- } \\
\text { bugging, system joint debugging }\end{array}$ \\
\hline & Debug content & $\begin{array}{l}\text { Circuit continuity, voltage status, operating point, circuit } \\
\text { function, }\end{array}$ \\
\hline & Circuit test & Static test, dynamic state, limit test \\
\hline \multirow{4}{*}{ Troubleshooting } & Fault type & \multirow{3}{*}{$\begin{array}{l}\text { Power supply and ground, instrumentation failure, circuit } \\
\text { connection, poor contact, device damage, impedance } \\
\text { Matching, electromagnetic interference }\end{array}$} \\
\hline & Failure analysis & \\
\hline & Troubleshooting & \\
\hline & $\begin{array}{l}\text { Troubleshooting } \\
\text { check analysis }\end{array}$ & $\begin{array}{l}\text { Power open circuit, short circuit, over current, frequency } \\
\text { range, over voltage, under voltage, power line voltage } \\
\text { drop , total Ground and grounding point ; Instrument } \\
\text { self-test, input and output impedance, coupling mode, } \\
\text { attenuation ratio, insurance Wire state, probe open circuit ; } \\
\text { Wiring error, not connected, disconnected cable, poor } \\
\text { contact, virtual Soldering; device damage } \\
\text {, component blown, capacitor short circuit ; Power supply } \\
\text { ripple, device noise, environmental drying Disturbance; } \\
\text { Inter-stage parameter coordination and distribution parame- }\end{array}$ \\
\hline \multirow{3}{*}{$\begin{array}{l}\text { Parameter } \\
\text { measurement }\end{array}$} & Instrument selection & Excitation source, frequency, amplitude, output imped- \\
\hline & Parameter Type & \multirow[b]{2}{*}{ Resistance measurement, capacitance measurement, in- } \\
\hline & Measuring circuit & \\
\hline
\end{tabular}




\begin{tabular}{|c|c|c|}
\hline & Measurement methods & $\begin{array}{l}\text { ductance measurement, DC voltage and current measure- } \\
\text { ment, AC voltage and current Measurement, waveform } \\
\text { measurement, high frequency signal, Q Value, gain, input } \\
\text { impedance, output impedance }\end{array}$ \\
\hline \multirow[t]{5}{*}{ data processing } & Form design & $\begin{array}{l}\text { Structural design, parameter selection ( direct, indirect ) } \\
\text { The data unit }\end{array}$ \\
\hline & data record & Digital selection, recording order \\
\hline & data analysis & Authenticity, rationality \\
\hline & data processing & $\begin{array}{l}\text { Calculation, processing ( average, denoising, etc. ), sort- } \\
\text { ing, display }\end{array}$ \\
\hline & Data representation & Data list, curve, histogram, pie chart, \\
\hline \multirow{5}{*}{ Error Analysis } & effective number & $\begin{array}{l}\text { The number of digits read by the instrument is selected, } \\
\text { and the number of digits in the data is selected. }\end{array}$ \\
\hline & Error category & Absolute error, relative error, reference error \\
\hline & Source analysis & \multirow{2}{*}{$\begin{array}{l}\text { Instrument accuracy, device parameter accuracy, observa- } \\
\text { tion error, contact resistance, power supply noise, line } \\
\text { division Cloth parameter loss, impedance matching, elec- } \\
\text { tromagnetic coupling interference }\end{array}$} \\
\hline & Error estimate & \\
\hline & Error elimination & $\begin{array}{l}\text { Correction, Substitution, Cancellation, Transform Range, } \\
\text { Average, Root Mean Square, Smoothing Filter, Bad Value } \\
\text { Eliminate }\end{array}$ \\
\hline \multirow[t]{8}{*}{ system design } & demand analysis & $\begin{array}{l}\text { Engineering social application background, technical } \\
\text { method research status, expected target function index , } \\
\text { application measurement Test method }\end{array}$ \\
\hline & $\begin{array}{l}\text { Environmental estab- } \\
\text { lishment }\end{array}$ & $\begin{array}{l}\text { Instrument equipment, device modules, processing and } \\
\text { production, } \\
\text { software tools, development environment, financial sup- }\end{array}$ \\
\hline & System planning & $\begin{array}{l}\text { Functional indicators, implementation methods, program } \\
\text { demonstrations ; System software and hardware structure, } \\
\text { module division, implementation Process; Project process, } \\
\text { manpower allocation }\end{array}$ \\
\hline & hardware design & Circuit design , component selection, simulation optimiza- \\
\hline & System implementa- & Software design, hardware installation, soldering, debug- \\
\hline & software design & $\begin{array}{l}\text { Standardization, generalization, modular design , commis- } \\
\text { sioning }\end{array}$ \\
\hline & System test & $\begin{array}{l}\text { Module function debugging, system joint debugging, func- } \\
\text { tional testing, indicator testing; Operability, stability Sex } \\
\text { and reliability testing; System Optimization }\end{array}$ \\
\hline & analysis Summary & $\begin{array}{l}\text { Implementation method design optimization,expected target } \\
\text { achievement,results expansion and promotion prospects }\end{array}$ \\
\hline
\end{tabular}




\begin{tabular}{l|l|l}
\hline Show speech & $\begin{array}{l}\text { Design summary report , exhibition board , PPT Design , } \\
\text { speech design }\end{array}$ \\
\hline
\end{tabular}

Table 5. Comprehensive quality

\begin{tabular}{|c|c|}
\hline $\begin{array}{l}\text { Table 4. Practical ability } \\
\text { Innovative thinking and }\end{array}$ & $\begin{array}{l}\text { Sensitivity,comprehension,creative idea,creative passio; Creativity,logic,deductive } \\
\text { reasoning }\end{array}$ \\
\hline $\begin{array}{l}\text { Literature search data anal- } \\
\text { ysis }\end{array}$ & Literature search, data retrieval, information statistics, comprehensive analysis \\
\hline $\begin{array}{l}\text { Problem analysis engineer- } \\
\text { ing modeling }\end{array}$ & Active learning, lifelong learning, a combination of theory and practice \\
\hline Program design evaluation & $\begin{array}{l}\text { Information resources, self-ability, knowledge structure, environmental condi- } \\
\text { tions, core issues, difficulties, }\end{array}$ \\
\hline $\begin{array}{l}\text { Knowledge integration } \\
\text { system design }\end{array}$ & $\begin{array}{l}\text { Self-learning, acquiring knowledge, flexible use, and fusion of knowledge meth- } \\
\text { ods }\end{array}$ \\
\hline $\begin{array}{l}\text { Software design simulation } \\
\text { optimization }\end{array}$ & $\begin{array}{l}\text { Theoretical derivation, modeling analysis, system design, simulation optimiza- } \\
\text { tion }\end{array}$ \\
\hline $\begin{array}{l}\text { Strive for resources to cre- } \\
\text { ate conditions }\end{array}$ & Query information, create conditions, build environments, get help, seek support \\
\hline $\begin{array}{l}\text { Engineering implementa- } \\
\text { tion of comprehensive test- } \\
\text { ing }\end{array}$ & $\begin{array}{l}\text { Project implementation, engineering implementation , functional commissioning, } \\
\text { performance testing }\end{array}$ \\
\hline $\begin{array}{l}\text { Comprehensive evaluation } \\
\text { of project performance }\end{array}$ & $\begin{array}{l}\text { R\&D, operational cost accounting, effectiveness, cost performance, reliability, } \\
\text { stability analysis }\end{array}$ \\
\hline $\begin{array}{l}\text { Integrated project manage- } \\
\text { ment }\end{array}$ & $\begin{array}{l}\text { Task division, target allocation, resource use, manpower allocation, process } \\
\text { scheduling }\end{array}$ \\
\hline $\begin{array}{l}\text { Teamwork communication } \\
\text { and coordination }\end{array}$ & $\begin{array}{l}\text { Organizational leadership, relationship coordination, academic exchange, } \\
\text { creating an atmosphere }\end{array}$ \\
\hline $\begin{array}{l}\text { System summary speech } \\
\text { expression }\end{array}$ & $\begin{array}{l}\text { Design summary, performance analysis, project outlook, written, language ex- } \\
\text { pression, self-presentation }\end{array}$ \\
\hline
\end{tabular}

\section{Comprehensive environmental requirements for experiments}

The Electrotechnics and electronics experiment teaching should fully meet the needs of students'information retrieval, self-study, research and exploration, design simulation, production test, summary and exchange, such as research, design, analysis, simulation, experiment, production, welding, test, extra-curricular research, subject competition and so on. Full openness should be achieved in time, space, content and resources. Students can study and practice independently anytime and anywhere, and can receive guidance, support and help ${ }^{[6]}$. In order to implement the basic standards of experimental teaching, the requirements for comprehensive environment construction, such as experimental teaching conditions, equipment conditions, teaching resources, operation mechanism, open management and information management, are put forward. 


\subsection{Requirements for experimental equipment and teaching resources.}

\subsubsection{Experimental teaching conditions}

The laboratory should have the functions of teaching demonstration, discussion speech, information inquiry, installation and welding, debugging and testing, writing reports and other teaching and practical forms.

\subsubsection{Experimental instruments and equipment}

The laboratory should be equipped with voltage source, digital multimeter, signal generator, digital oscilloscope and other general experimental instruments, as well as logic analyzer, frequency sweeper, transistor tester, bridge, RCL tester, virtual instrument and other special equipment. And equipped with electronic circuits, alternating current circuits, FPGA/CPLD, microcontroller/embedded systems, and electronic circuits, alternating current circuit design, analysis, simulation, debugging and production of software tools, temperature, humidity, illumination, weight, sound, speed, angle and displacement as the object of measurement or Control objects, etc.

\subsubsection{Requirements for teaching resources}

The experimental center should construct a website of experimental teaching information resources which is often updated to provide teaching contents, curriculum arrangement, electronic teaching plans, teaching videos, knowledge and methods, technical programs, engineering cases and other auxiliary teaching resources.

\subsection{The operation and management mechanism of experimental teaching.}

\subsubsection{Laboratory Configuration}

The experimental center should set up comprehensive laboratories at different levels, such as functional basis, professional basis and professional level, so as to improve the utilization of space and equipment resources.

\subsubsection{Operation and management system}

Establish the maintenance and maintenance system of instruments and equipment to ensure the equipment intact rate; establish a variety of post responsibility system to ensure the order of experimental teaching.

\subsubsection{Open management mechanism}

There should be plenty of open time and open space; there should be open management mechanism such as reservation, assignment, login, withdrawal, and so on; there should be the use mechanism of instruments and equipment, experimental platform, components and other resources suitable for personalized experiments.

\subsubsection{Safety guarantee mechanism}

Establish safety and accident emergency handling mechanism, equipped with security, fire fighting facilities and accident handling supplies.

\subsection{Information management in experimental teaching}

In view of open individualized experiment teaching, information aided teaching is adopted.

With the process management system, it should cover the implementation of the curriculum organization plan, the overall arrangement of the teaching process, the statistics of the examination and evaluation results, the multiple channels of interaction between teachers and students, the management of appointment and assignment, the approval and issuance of device applications, the collection of experimental information on the spot, the submission and correction of electronic reports, the management of the process of practical projects, and the test questions. Educational management and quality assurance functions such as volume survey, equipment and equipment management.

\section{Implementation of research results}

\subsection{The basic standards of electrical and electronic experimental teaching are applied and tested in the schools of the research group members first}

The Electrotechnics and electronics experimental center of Southeast University aims at cultivating students'abilities of independent research, knowledge synthesis, innovative design and engineering realization. It focuses on improving the quality of research and exploration, innovative thinking, communication and teamwork. It reforms the innovative curriculum system, carefully designs experimental projects, optimizes the teaching process and integrates them. To 
create a practical environment, to create an information management system, to create both innovation, practicality and popularization, highlighting the independent study of electrical and electronic practice courses. The construction of the project has broken the situation that many electrician and electronics experimental courses are independent of each other and difficult to carry out comprehensive engineering practice; solved the problems of simple experimental verification knowledge, students'self-study, analysis and research pressure and space shortage; solved the problems of direct teaching in the past, inadequate analysis and guidance, task gradient and task gradient. There are many problems such as the unity of levels and the lack of teaching students in accordance with their aptitude. In the course of teaching practice, curriculum reform and teachers try to change propositions and conditions to guide students to explore, construct knowledge and understand rules independently and cultivate students'autonomous learning ability. Let students understand the functions and principles of the system.

\subsection{Appropriate variants, promote divergence}

The purpose of variants is to guide students to explore physical problems from various angles. The experimental design aims to guide students from the "changeable" physical quantity and conditions to discover the essence of "unchangeable" physical laws, explore its principles and laws from the "unchangeable" nature, cultivate students'divergent thinking and develop their creativity.

\subsection{Problem Leadership, Enlightenment Wisdom}

Expanding the problem around the construction of binary phase shift keying frequency band transmission system model, meticulous questioning, flexible questioning, stimulate students'enthusiasm for inquiry, cultivate students' thinking quality, guide students to grasp research and solve the problem of multiple strategies for students to build a scaffold for thinking, Let students build their own knowledge. Under the guidance of the problem, students carefully observe the simulation results, start thinking to a deeper level, the ability to improve.

\section{Concluding remarks}

Through the combination of software simulation and theory in the digital signal frequency band transmission experiment, the students'enthusiasm and initiative are aroused, so that they can grasp the relevant concepts and principles more systematically and profoundly. Simulation technology has changed the form and content of experimental teaching of communication principle course, made up for the deficiency of traditional experimental teaching in teaching means, provided abundant perceptual materials, and made abstract physical laws more visualized. Practical ability has been trained, and remarkable teaching results have been achieved. At the same time, it has laid a good foundation for students to continue to work and study in relevant professional fields.

\section{References}

1. Wang WT, Zhao JC, Gu ZF, et al. Application of LabVIEW in the Teaching of Basic Circuit Analysis Course [J] Experimental Science and Technology, 2014, 12 (3): forty nine to one.

2. Chen J. Application of System View in Experimental Teaching of Communication Principles Course [J] Journal of Heze University, 2015 (2): 101-105.

3. Wang F. Application of computer simulation in experimental teaching of communication principle[J] experimental science and technology, 2014, 12 (4):

4. Zou D SystemView in Modern Communication Principles Course [J]. Journal of East China Jiaotong University, 2007, 24 (B12): 51-53.

5. Chen J. Model Design and Analysis of Digital Baseband Transmission System Based on System View [J] Laboratory Research and Exploration, 2013, 32 (10): 98-101.

6. Ren J, Zhang HY. Reform of Communication Principle Experiment Teaching [J] by Virtual Simulation Experiment. Experimental Technology and Management, 2014, 31 (3): 95-97.

7. Chen J. Teaching Practice of Communication Principles Based on System View [J] Physical Experiment, 2014,34 (8): 20-24.

8. Chen J. Research on Simulation Experiment Teaching of Communication Principle Course Based on System View [J]. Journal of Wuzhou University, 2014 (6): 79-85.

9. Jiang YL, Liu LF. Activating Students'Subjective Consciousness and Developing Students' Autonomous Learning Ability [J]. China Audiovisual Education, 2007 (12): 97-98. 
10. Chen J. Application of System View in experimental teaching of communication theory course[J] automation and instrumentation, 2015 (2): 2010 - 2010.

11. Chen J. The Integration of Modern Educational Technology and Physics Teaching: Using "Geometry Sketchpad" to Assist and Optimize Physics Teaching Process [J] Journal of Kashi Normal University, 2009, 30 (3): 88-91.

12. Chen J, Yu C, Wei XS, et al. Examining the Integration of MCAI and High School Physics Teaching from the Perspective of Modern Educational Technology [J]. Journal of Gansu Normal University, 2010, 15 (2): 84-87.

13. Sun Y, Dai YF. System View Communication Simulation Development Manual [M]. Beijing: National Defense Industry Press, 2004:100-120.

14. Gao Xiquan, Ding Yumei. Digital Signal Processing [M] 3. Xi'an: Xi'an University of Electronic Science and Technology Press, 2009:130-136.

15. Fan CX. Communication Principles [M] 5. Beijing: National Defense Industry Press, 2003:151-160. 